are discussed. The same method of considering the given equation as the limiting case of a system of algebraic equations is used in treating this type. The kernel is supposed to be finite. The case where the kernel becomes infinite is discussed very briefly. A few pages are devoted to systems of equations and equations involving multiple integrals. At the end of the chapter some very interesting applications are made to Dirichlet's problem and to the vibration of strings.

In Chapter IV a very brief and incomplete account of integro-differential equations is given. By such an equation is meant one involving not only the unknown functions under signs of integration but also the unknown functions themselves and their derivatives. No attempt is made to give a systematic treatment of this subject. A few problems from mechanics leading to equations of this kind are discussed. A few pages are also devoted to permutable functions. For a more complete discussion of these very interesting topics the reader is referred to papers by Volterra published in Acta Mathematica and Atti d. R. Accademia dei Lincei. It is with great pleasure that we receive the news that the author intends to give an exhaustive treatment of these topics in a second volume which will soon be published.

\title{
JACOB WestLUND.
}

Die komplexen Veränderlichen und ihre Funktionen. Von Dr. Gerhard Kowalewski, Ord. Professor an der Hochschule zu Prag. Leipzig und Berlin, B. G. Teubner, 1911. $455 \mathrm{pp}$.

This volume, by the well-known author of the recently published text on determinants, is intended to be a continuation of the Grundzüge der Differential- und Integralrechnung, which was reviewed on page 531 of volume 19 of the Bulletin, as well as an introduction to the theory of functions. Some of the very convenient terms introduced in the aforementioned book, such as the expression "fast alle," meaning "all with a finite number of exceptions," so useful in the discussion of propositions involving the limits of sequences, are also used in this book. Other new ones are introduced, as for instance the "Hof" of a point, meaning a circle having the given point as a center. This strikingly descriptive terminology, as well as the interjection in appropriate places 
of interesting historical remarks,* lend to the style of this author a certain charm and clearness which it is difficult to characterize more specifically, but which must be evident to any one who reads his books; among these I should like to mention his Klassische Probleme der Analysis des Unendlichen, a book that seems to the present reviewer to be well worth the attention of teachers of the calculus. Professor Kowalewski succeeds in a remarkable way in bringing at least some features of the more advanced portions of analysis within the reach of the less advanced student; this gives his books added pedagogical value, inasmuch as they arouse the reader's interest in the regions lying a little beyond his present capacity.

The text on the complex variable and its functions possesses all the good qualities of the former productions of this author. Let me say at the outset that the book treats only of uniform functions, so that with the exception of a brief passage on page 319 , no mention is made of a Riemann surface nor of any of the subjects connected therewith. The book is divided into seven chapters, the first one of which, comprising 64 pages, deals with the representation of complex numbers by means of the points of the plane, and with a discussion of the general linear fractional function of the complex variable $z$. In addition to the topics usually treated under this heading, the author gives in this chapter: first, a treatment of the transformation $z^{\prime}=(a \bar{z}+b) /(c \bar{z}+d)$, where $\bar{z}$ is the conjugate of $z$; this section closes with a proof of the fact that all the elementary transformations, including rotations and translations, can be compounded out of reflections; second, a treatment of the linear fractional function in the homogeneous form, which gives an opportunity to bring in some interesting facts about Hermitian forms. The classification of finite linear transformation groups, including the modular group, leads to a digression on the equivalence of positive quadratic forms and to a short study of linear and planar "Punktgitter," useful later on in the discussion of doubly periodic functions.

Chapter II, 18 pages, is devoted to complex functions of a

\footnotetext{
* Such as, for instance, the remark on p. 91 of the present volume, just before a proof of the fundamental theorem of algebra, that Leibniz did not believe it to be possible to solve the equation $z^{4}+1=0$ by the aid of complex numbers.
} 
real variable. The first and second mean-value theorems of the integral calculus are proved with the utmost rigor by means of the notion of the "smallest convex enclosure" of a given point set $M$. By this is meant the set consisting of all the points common to all the convex sets which contain $M$. It is proved that whenever $M$ is closed and connected, then its smallest convex enclosure consists of all the points which lie on chords of $M$.

In Chapter III comes the introduction of the analytic or monogenic function, defined as a function for which the derivative exists. The definition of the derivative is given in a beautifully clear but at the same time completely rigorous way; the term " ausgezeichnete Folge," used so successfully in the "Grundzüge," and the theorem that all sub-sequences of a given convergent sequence are convergent, and that their limit is the same as that of the original sequence, applied to great advantage throughout the book, help materially in accomplishing this result. The analytic character of rational functions, the fundamental theorem of algebra, the unlimited differentiability and integrability of power series in the interior of their circles of convergence are proved in the first 26 pages of this chapter. The remaining 25 pages are given up to the exponential and trigonometric functions, defined by means of power series, and to the inversion of the former, the logarithmic function, closing with a section on functions of a function. The Mercator map is introduced by way of the conformal representation of a cylinder upon a circular ring. In the discussion of the logarithmic function, the different determinations are kept distinct and treated as different functions; it is shown that each one of them is determined by its derivative and by its value at one point. The expansions of the principal logarithms of $1+z$ and of $(1-z) /(1+z)$ lead very naturally to a discussion of series of the form

$$
\sum_{n=1}^{\infty}(-1)^{n-1} \frac{\cos n \varphi}{n^{p}} \text { and } \sum_{n=1}^{\infty}(-1)^{n-1} \frac{\sin n \varphi}{n^{p}} \quad(p=1,2, \cdots) .
$$

Chapter IV, entitled "Curvilinear integrals," contains within its 46 pages propositions relating to the integrals of functions of a complex variable along rectifiable curves. The existence of 


$$
\int_{A B} f(z) d z
$$

is proved for a continuous function $f(z)$ by reducing to integrals of functions of a real variable, and also directly without separating into real and imaginary parts. It would take us too far afield to report in detail upon the mode of presentation, which is a model of clearness and rigor. For the fundamental theorem that a function which has the derivative $f(z)$ at every point of a path $A B$ is representable in the form

$$
C+\int_{A B} f(z) d z
$$

the path of integration is further conditioned so as to have the following property: If $z_{1}, z_{2}, \cdots$ is a sequence of points on $A B$, converging to a point $z_{0}$ of $A B$, but not containing $z_{0}$, and if $l_{n}$ is the length of the path $z_{0} z_{n}$, then $\lim l_{n} /\left|z_{0}-z_{n}\right|$ must exist and be finite. This condition is equivalent to the one used by Moore in his proof of the Cauchy-Goursat theorem (see Transactions of the American Mathematical Society, volume 1), but seems to be simpler in form. All paths of integration considered from here on are assumed as having this property. Formulas are obtained for the coefficients of a power series in the usual way and also by means of Hadamard's formulas in terms of the real and imaginary parts of $f(z)$ expressed in terms of polar coordinates. The latter results are then used to derive Poisson's integral.

Chapter V is devoted to Cauchy's fundamental theorem and its consequences. It covers 143 pages and is the longest single chapter in the book. Cauchy's fundamental theorem is given first for a rectangle, by the method of Moore. A little farther on follow two proofs of the same fundamental theorem for so-called " Normalbereiche," defined as the set of points given by the formula

$z=\left\{u+i(1-t) \psi_{0}(u)+i t \psi_{1}(u)\right\} e^{i \gamma} \quad(a \leqq u \leqq b, 0 \leqq t \leqq 1)$,

where $\psi_{0}(u)$ and $\psi_{1}(u)$ are real and continuous functions of limited variation on the interval $(a b)$, subject to the restriction $\psi_{0}(u)<\psi_{1}(u)$. The first of these proofs proceeds by means of decomposition into rectangles; the second one proceeds directly by a generalization of the method used for the 
case of a rectangle. By the same method again, Green's theorem is proved for two functions $u(x, y)$ and $v(x, y)$, which are assumed to be "properly differentiable." A function $f(x, y)$ is said to be properly differentiable at a point $\left(x_{0}, y_{0}\right)$, if there exist two functions $f_{1}(x, y)$ and $f_{2}(x, y)$ such that

$\lim \frac{f\left(x_{n}, y_{n}\right)-f\left(x_{0}, y_{0}\right)-\left(x_{n}-x_{0}\right) f_{1}\left(x_{0}, y_{0}\right)-\left(y_{n}-y_{0}\right) f_{2}\left(x_{0}, y_{0}\right)}{\left|x_{n}-x_{0}\right|+\left|y_{n}-y_{0}\right|}=0$,

whenever $\lim x_{n}=x_{0}$ and $\lim y_{n}=y_{0},\left(x_{n}, y_{n} \neq x_{0}, y_{0}\right)$.

From this it follows that the function $f(z)$ possesses a derivative at the point $z_{0}$, when and only when its real and imaginary parts $u$ and $v$ are properly differentiable at $\left(x_{0}, y_{0}\right)$ and satisfy the conditions

$$
\frac{\partial u}{\partial x}=\frac{\partial v}{\partial y}, \quad \frac{\partial u}{\partial y}=-\frac{\partial v}{\partial x} .
$$

This result seems distinctly valuable inasmuch as it states the exact condition under which the two definitions of an analytic function are equivalent. Next come, very much as is customary, the important consequences of Cauchy's theorem: Cauchy's formula, Taylor's series, Laurent's series; followed by Liouville's theorem, the theorems on rational functions and residues, the second proof of the fundamental theorem, etc. The author proposes the term "Laurent point " of a function for a point at which the function is either regular or has an isolated singular point, i. e. a point at which the function may be developed into a Laurent series, a regular point then becoming a "regular Laurent point." The use of the proposed term throughout the rest of the book is a good argument in favor of its acceptance, for it enables the author frequently to simplify his statements. Between the Taylor series and the Laurent series, 15 pages are devoted to boundary-value problems. There is a clear treatment of the two-dimensional boundary-value problem for the circle and of the reduction of this problem and of the corresponding one for the ellipse to linear integral equations. This is one of those instances in which the author has brought the more advanced topics of his subject within the scope of a more elementary text. The last 34 pages of the chapter are devoted to the inversion of a system of two functions $u(x, y)$ and $v(x, y)$ and to the inversion of power series in the neighborhood of 
simple and of multiple zeros of the function. The discussion of the latter case is the only place in the book where, as noticed above, a Riemann surface is mentioned. Conformal representation gets an admirable discussion in connection with this subject by means of the notion of a "Jordan neighborhood" of a point $\left(x_{0}, y_{0}\right)$, by which is meant the totality of all points in the interior of a Jordan curve which surrounds the point $\left(x_{0}, y_{0}\right)$. This chapter closes with the discussion of the transformation effected by $w=f(z)$ in the neighborhood of a multiple zero.

Chapters VI and VII take up the subjects of infinite series and infinite products of functions of a complex variable. The definitions and well-known theorems on uniform and absoluteuniform convergence are followed in section 68 by the definition of " normal convergence," as given by Baire in his Leçons sur les Théories générales de l'Analyse. Then comes the theorem that every uniformly convergent series can be transformed into a normally convergent series by the omission of a finite number of terms at the beginning of the series and by grouping of neighboring terms. No reference is given for this theorem; it seems to be due to Baire, who proves it on page 30 of volume 2 of the book, mentioned above. The complete absence from the text under review of references to the literature is unfortunate. Creating, as it cannot help doing, a great deal of interest in allied fields of mathematics, the book loses a great deal of its value by not giving the reader any guidance in his attempts to gain further knowledge of these fields. The theorem to which reference was made above and the concept of normal convergence, including as it does uniform and absolute-uniform convergence, prove to be very useful in several of the later proofs on infinite series and infinite products. The discussion on pages 348 and 349 of the derivatives of a uniformly convergent series of differentiable functions seems to be unnecessarily lengthened by the proof of the formulas for the successive derivatives of the function $s(z)$; as soon as the existence of the first derivative is proved, these results can be obtained by application of the results of pages 215 and 216. The theorem of Heine-Borel (or rather that of Borel-Heine, as the author calls it with a regard for historical accuracy, not marred by national prejudice) is used to prove the following theorem: If $P$ is a point set which contains interior points, if $f_{n}(z)$ are functions which are differenti- 
able at every interior point of $P$, and if the series $\Sigma f_{n}(z)$ is uniformly convergent on every closed subset of $P$, which contains interior points only, then this series may be differentiated term by term indefinitely at every interior point and the resulting series converge uniformly on every such subset.

Nearly one half of these last two chapters, 64 pages, is given to doubly periodic functions and to elliptic integrals. The book ends with the presentation of the theorem of Mittag-Leffler and of Weierstrass's theorem on the factorization of entire functions. It is surprising that the subject of analytic continuation does not even receive mention.

The misprints are rather more numerous than is usual in Teubner's books, but they are not very important. It will suffice to point out the following:

Page 95: line 16 from top: read $R(z)$ instead of $Q(z)$.

Page 119: line 8 from bottom: read $F(\omega)$ instead of $G(\omega)$.

Page 129: line 6 from top: read $\frac{1}{2^{3}}+\frac{1}{4^{3}}+\frac{1}{6^{3}}+\cdots$ instead

$$
\text { of } \frac{1}{2^{3}}+\frac{1}{3^{3}}+\frac{1}{4^{3}}+\cdots \text {. }
$$

Page 284: line 1 from bottom: read $\nu^{\prime}+\nu^{\prime \prime}=\omega^{\prime}+\omega^{\prime \prime}$ instead of: $\nu^{\prime}=\nu^{\prime \prime}-\omega^{\prime}+\omega^{\prime \prime}$.

Page 349: lines 1 and 6 from top: the last exponent on each of these lines should read $-(p+2)$ instead of $-(p+1)$.

line 10 from top: a factor $M \rho$ should be multiplied into the second term on the right-hand side of the equation.

Page 375: line 3 from top: read $2 \pi$ instead of $\pi / 2$.

Arnold Dresden.

Calcul des Probabilités. Par Louis Bachelier. Tome I. Paris, Gauthier-Villars, 1912 . vii $+516 \mathrm{pp}$. Price $25 \mathrm{fr}$.

THE object of this book is to give not merely an exposition of some of the leading principles long known in the theory, but to present recent methods and results, due to the author, that represent from certain points of view a decided transformation of the calculus of probabilities.

The conception of continuous probabilities is at the foundation of this change. The author points out the fact that the 